\title{
Effects of Phenobarbital on Seizure Activity in the Gerbil
}

\author{
KATHY S. WATANABE, ${ }^{(15)}$ RICHARD J. SCHAIN, AND BEVA G. BAILEY \\ Departments of Pediatrics, Neurology and Psychiatry, Neuropsychiatric Institute, University of California, Center for \\ the Health Sciences, Los Angeles, California, USA
}

\section{Summary}

This study was concerned with the effect of chronic phenobarbital treatment of seizure behavior in a strain of Mongolian gerbils (Meriones unguiculatus) that is predisposed to spontaneous seizures. Subcutaneous injections of $60 \mathrm{mg} / \mathrm{kg}$ phenobarbital were given daily to the experimental animals from 1-5 months of age. Control animals received equivalent doses of vehicle. At the termination of treatment (5 months), animals were tested regularly for seizure susceptibility until 1 year of age. Phenobarbital-treated animals exhibited an intensification of seizure activity both during and after the period of drug treatment. All animals receiving phenobarbital had one or more spontaneous seizures during the treatment period; $67 \%$ of the controls exhibited seizures. During this time phenobarbital-treated animals had an average of $\mathbf{1 0 . 5}$ seizures with a mean seizure degree of +3.2 (scale of 1-5), whereas the controls had an average of 3.5 seizures with a mean seizure degree of +2.4 . During the entire 7-month post-treatment period, the phenobarbital-treated animals displayed a mean seizure degree of +3.4 as opposed to +2.3 for the controls; drug-treated gerbils exhibited more frequent unstimulated seizures $(66 \%)$ than did controls (37\%). The latency to seize was shorter for the phenobarbital-treated animals $(142 \mathrm{sec})$ than for controls $(233 \mathrm{sec})$, and experimental animals had seizures of greater duration (231 sec) than did controls (107 sec). (All comparisons are significant at the 0.001 level.) These studies reveal that chronic phenobarbital administration enhances seizure activity in the gerbil. This enhancement persists long after termination of phenobarbital treatment. The explanation for this paradoxical effect may lie in the effects of phenobarbital upon the development of brain inhibitory mechanisms.

\section{Speculation}

Phenobarbital has been shown to function as both a convulsant and an anticonvulsant agent when administered under differing conditions to seizure-sensitive Mongolian gerbils. It would seem that the timing and/or duration of administration of the drug may determine the ultimate effect of phenobarbital on the central nervous system. It may be that this paradoxical effect is also obtained in other species.

Phenobarbital is the most commonly used anticonvulsant agent in humans. Its popularity stems from its relative freedom from adverse side effects on major organ systems (7). However, there have been virtually no studies of its neurophysiologic effects when given over prolonged periods to immature animals. Recent work in the laboratory has revealed that chronically administered phenobarbital will retard brain growth in preweanling rats $(2,10,11)$. In order to correlate anticonvulsant and biologic effects of phenobarbital, we initiated a series of studies on the effects of administration of this drug to the Mongolian gerbil (M. unguiculatus), an animal predisposed to exhibit "spontaneous" seizures (9). These seizures appear to be a form of reflex epilepsy, as they are precipitated by changes in the environment or exogenous stimuli.

\section{MATERIALS AND METHODS}

The progeny of Mongolian gerbils ( $M$. unguiculatus), bred for seizure-sensitivity (12), were weaned at 30 days of age. Animals within each litter were randomly allocated to control and phenobarbital groups. An attempt was made to equalize within a litter the number of animals of one sex receiving a particular treatment, and to balance overall the number of males and females in each treatment group (18 control males, 13 phenobarbital-treated males; 15 control females, 12 phenobarbital-treated females.) The animals were segregated according to sex, but control and phenobarbital-treated animals within a litter were otherwise housed together.

Our previous experience with developing rats indicated that a dose of $60 \mathrm{mg} / \mathrm{kg}$ did not produce excessive serum levels and was easily tolerated for prolonged time periods after adaptation to initial sedative effects $(2,11)$. To determine whether this dose level would be an effective anticonvulsant in the gerbil, 12 adult gerbils with reliable seizure histories were subjected to daily sc injections of $60 \mathrm{mg} / \mathrm{kg}$ phenobarbital for 5 days. A similar group of 12 gerbils received vehicle for the same period. All animals were tested for seizure capability $4 \mathrm{hr}$ after the final injection.

Seizures were scored on a scale of $0-5$ (9) $(5=$ maximal seizure), with the addition of intermediate values (i.e., a 2.5 seizure was judged to be distinct from a 2.0 or 3.0 seizure and so on).

For the determination of the effects of chronic phenobarbital administration, sc injections of $60 \mathrm{mg} / \mathrm{kg}$ phenobarbital were administered 5 days weekly to the experimental animals from $1-5$ months of age. Control animals received equivalent doses of vehicle. Animals were not tested for seizure activity during the period of drug treatment, but seizures were frequently elicited by the normal handling procedures involved in weighing the animals and administering the drug. Such seizures were termed "spontaneous" to differentiate them from seizures observed during a test interval. Upon termination of phenobarbital administration, animals were tested weekly for occurrence of seizures in the test situation. Each animal was placed individually in a large pan and allowed to explore it freely for $5 \mathrm{~min}$. If a seizure occurred within this space of time, it was termed an "unstimulated" seizure and the degree of severity, latency, and duration were noted. If, at the termination of the free-running period, no seizure had occurred, the animal was stressed by rapidly and persistently stroking its back, eliciting an escape response. The animal was stroked for a maximum of $2 \mathrm{~min}$. A seizure response obtained during stimulation was noted as a "stimulated" seizure and the degree of severity, latency, and duration were recorded. The latency period always included the $5 \mathrm{~min}$ of free-running activity.

Tests were at weekly intervals for the first six testings and then at monthly intervals until the animals were a year old. They were then killed, the brains quickly dissected out, weighed, frozen in liquid $\mathrm{N}_{2}$ and stored at $-70^{\circ}$ for later biochemical analyses. 
The serum half-life of phenobarbital was determined in 30- to 31-day-old gerbils. The animals were injected sc with $60 \mathrm{mg} / \mathrm{kg}$ phenobarbital and decapitated at discrete intervals after the injection. Blood was collected from the neck wound and pooled (four to six animals for each serum sample).

\section{RESULTS}

Administration of $60 \mathrm{mg} / \mathrm{kg}$ phenobarbital sc to gerbils produces a temporary period of sedation. The animals recover after a period of hours, the duration of which is longer for younger and naive animals. Feeding patterns are subsequently resumed, as evidenced by normal weight gain of drug-treated animals (Fig. 1).

A dosage of $60 \mathrm{mg} / \mathrm{kg}$ phenobarbital administered daily for a 5 -day period conferred anticonvulsant protection in adult gerbils (6-24 months of age). Ten of 12 gerbils with reliable seizure histories and mean seizure performance of +4 failed to seize when tested $4 \mathrm{hr}$ after the final phenobarbital injection (the two seizing animals displayed a mean seizure degree of +0.5 , and both of the seizures were elicited with stimulation). In contrast, of 12 control gerbils, $10(83.3 \%)$ displayed seizures on testing (mean degree of +4.2 ) and 3 of these were unstimulated. It can be seen that phenobarbital at this dosage is an effective anticonvulsant in the adult gerbil when administered over a short period of time.

In contrast, phenobarbital did not exert anticonvulsant effects when administered chronically from 1-5 months of age. Instead, intensification of seizure activity was observed both during the treatment period and after its termination. During the period of phenobarbital administration, phenobarbital-treated animals had an average of 10.5 seizures as opposed to only 3.5 for the controls, a 3-fold difference. Moreover, the animals receiving phenobarbital averaged seizures with an intensity of +3.2 whereas the controls exhibited a mean seizure intensity of $+2.4(P<0.001$ for both comparisons) (Fig. 2). All 25 of the phenobarbital-treated animals

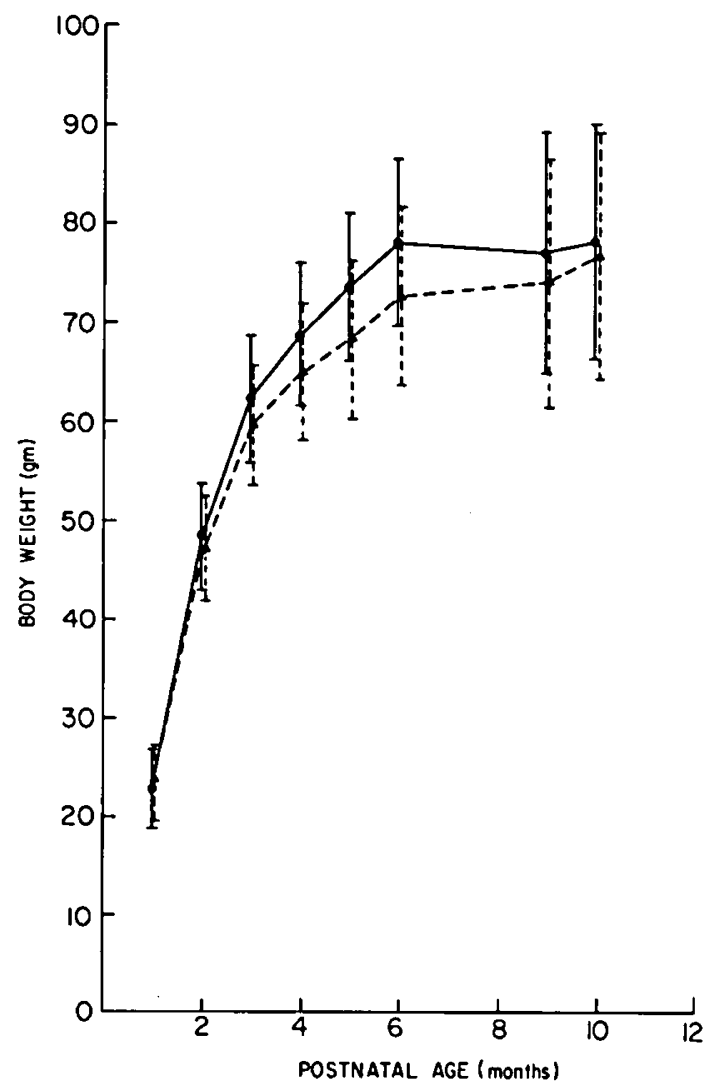

Fig. 1. Growth curves of control - $(n=33)$ and phenobarbitaltreated $\Delta---\Delta(n=25)$ gerbils during the period of injection and testing. (The points represent means and the bars are 1 SD on either side of the mean.)

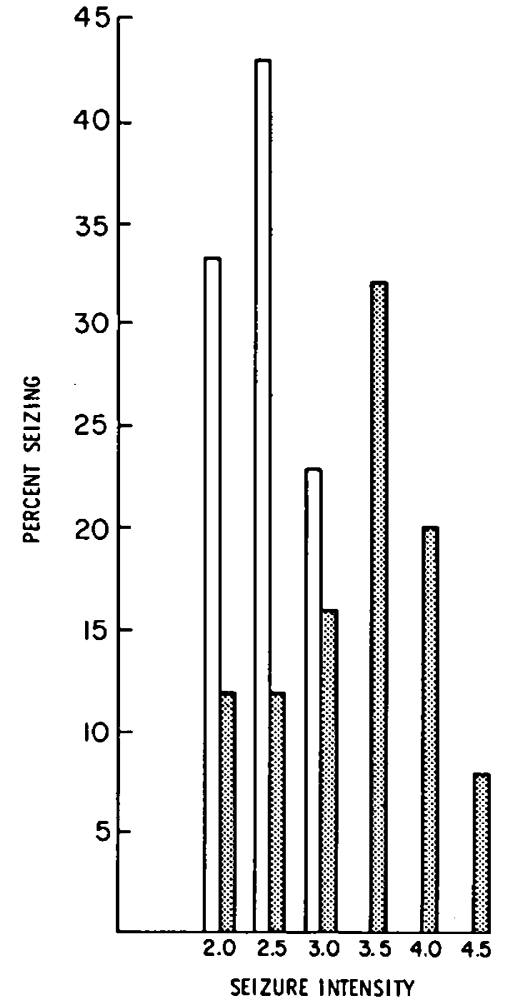

Fig. 2. Mean intensity of seizures observed during the 4-month period of phenobarbital administration. Values plotted as percentage of all animals exhibiting seizures. Percentages are based on means for each animal. Mean seizure intensity values were grouped for calculation of percentages. Each point represents a range of 0.4 seizure intensity units $(0.2$ units to either side). Control, $\square$; phenobarbital, 图.

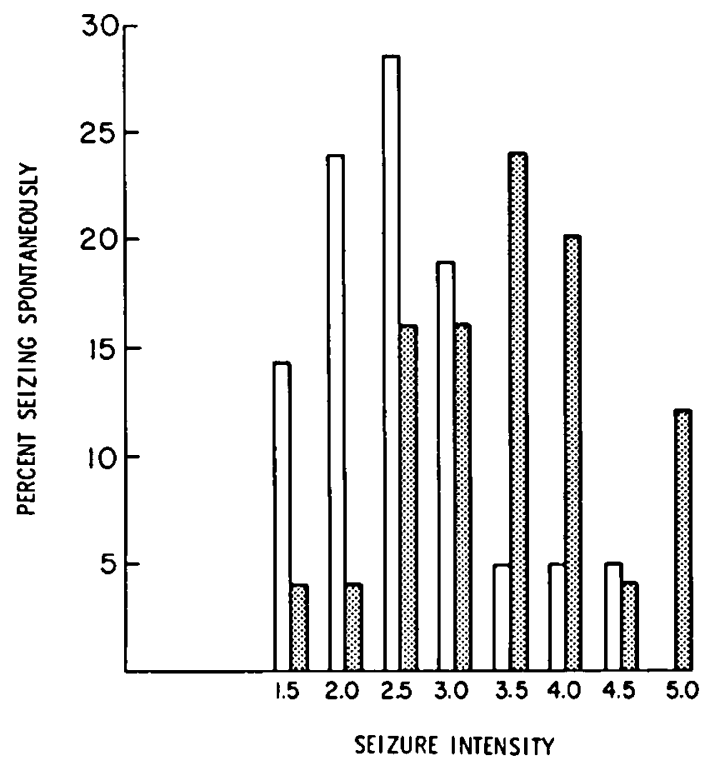

Fig. 3. Mean intensity of seizures obtained during the 7-month postphenobarbital treatment period. Values plotted as the percentage of all animals tested (including those that failed to produce seizures). Percentages are based on means for each animal. Mean seizure intensity values were grouped for calculation of percentages. Each point represents a range of 0.4 seizure intensity units ( 0.2 units to either side). Control, $\square$; phenobar bital, 四.

exhibited one or more seizures (spontaneous) during the treatment period; $67 \%$ of the controls (22 of 33 ) did so.

Throughout the entire 7-month postinjection period, phenobarbital-treated gerbils exhibited more intense seizures than did the 
controls. The mean seizure degree observed for the phenobarbital group was +3.4 , as opposed to +2.3 for the crintrols $(P<0.001)$ (Fig. 3). Other aspects of the seizure were significantly different for the two groups. Phenobarbital-treated gerbils exhibited more frequent spontaneous and unstimulated seizures than did control animals during and after the period of drug administration. (Fig. $4, a$ and $b$ ). Over all testings (postinjection period), the phenobarbital group averaged $66 \%$ unstimulated seizures compared to a mean of $37 \%$ for the control group $(P<0.001)$. The latency to seize was shorter for the phenobarbital animals $(142 \mathrm{sec})$ than it was for the controls $(233 \mathrm{sec})(P<0.001)$. See Figure 5 . In addition, the seizures were of greater duration for the phenobarbital animals $(231 \mathrm{sec})$ than for the controls $(107 \mathrm{sec})(P<0.001)$. See Figure 6.

Since the animals were not injected on the weekends, it was initially suspected that drug withdrawal may have occurred on a regular basis during the brief interval between treatments, and that this might account for the intensification of seizure activity observed among phenobarbital-treated animals during the entire treatment period. However, an analysis of the recorded seizures revealed no evidence of increased seizing on Mondays.

To determine the serum levels of phenobarbital at various intervals within a 24-hr period, $60 \mathrm{mg} / \mathrm{kg}$ phenobarbital was administered sc to previously untreated 30 -day-old gerbils (Fig.
7). The maximum serum level $(50 \mu \mathrm{g} / \mathrm{ml})$ was reached within an hour after the injections. By $24 \mathrm{hr}$ postinjection, it had dropped to $18 \mu \mathrm{g} / \mathrm{ml}$. The half-life was calculated on the basis of eight measurements to be $14.5 \mathrm{hr}(13)$.

To exclude the possibility that intensified postinjection seizures in the phenobarbital-treated animals might be due to withdrawal from the drug rather than due to a genuine effect, the gerbils were tested for seizure susceptibility for 6-7 months after termination of the treatment. After an initial 6 weeks of weekly testing, during which withdrawal effects might have influenced the results, the animals were checked on a monthly basis. An analysis of the monthly testings yielded equally significant findings: mean seizure degree for phenobarbital animals was +3.6 and for control animals, $+2.5(P<0.001)$. It is clear that the effect of phenobarbital on seizure activity is not a transient one.

Forty-nine percent ( 39 of 79) of the phenobarbital-treated gerbils died sometime during the treatment period as compared to $34 \%$ of the controls ( 23 of 67 animals). The experimental animals died at an average age of 44.1 days (range of 34-62 days) and controls at 42.7 days (range of 32-53 days). The rather high overall mortality rate was attributed to a severe infection of Tyzzer's disease (Bacillus pilliformis) which had its highest mortality among the colony weanlings. The higher death rate among the experimentals is probably due primarily to a drug-induced weakness
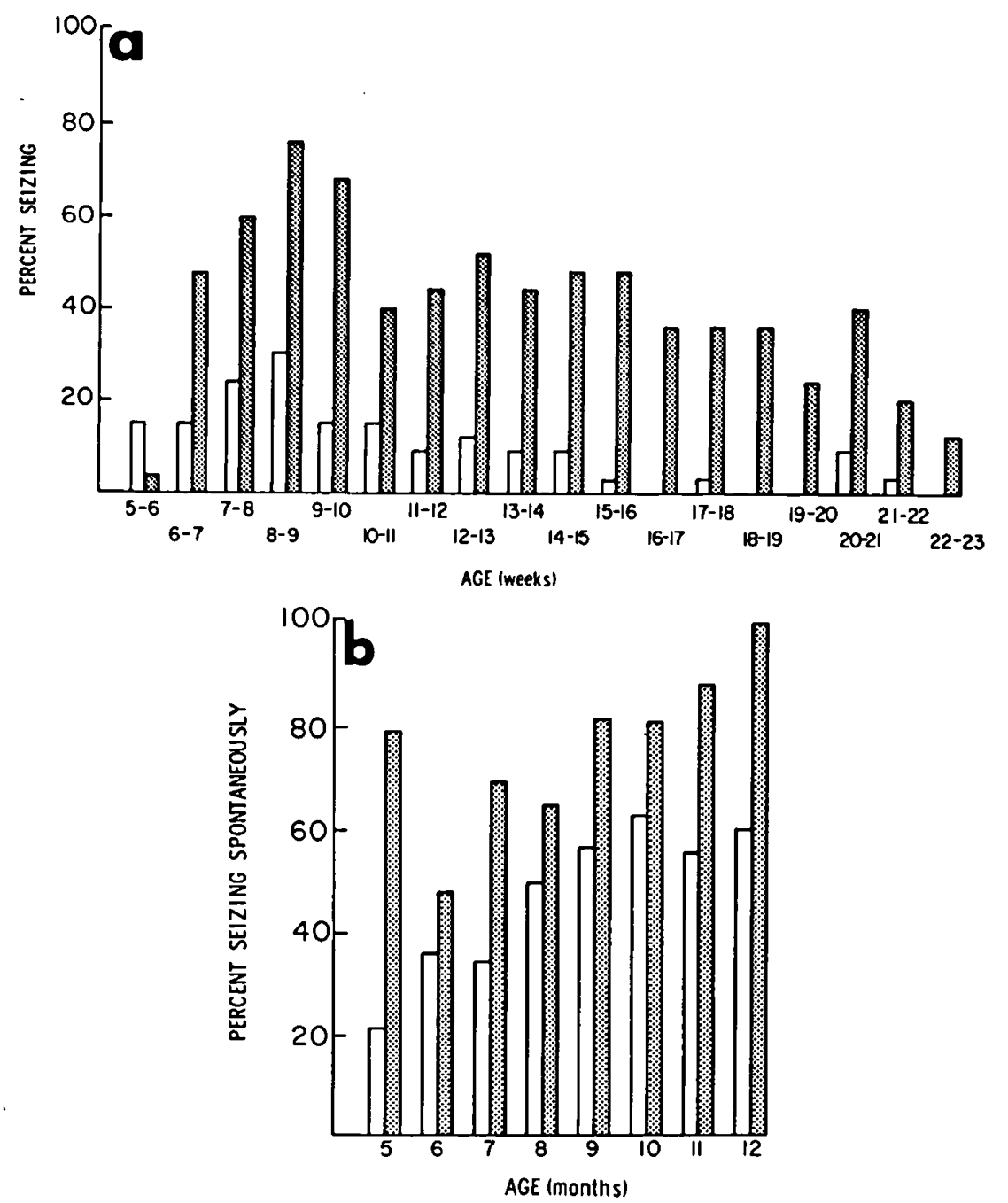

Fig. 4. $a$ : percentage of control or phenobarbital-treated animals that exhibited one or more seizures during successive 1-week intervals (during the 4-month phenobarbital treatment period). Control, $\square$, phenobarbital, $\square$. b: percentage of control or phenobarbital-treated gerbils that exhibited unstimulated seizures during the 7-month period after termination of phenobarbital administration. Animals were tested at monthly intervals. Control, $\square$; phenobarbital, $\square$. 


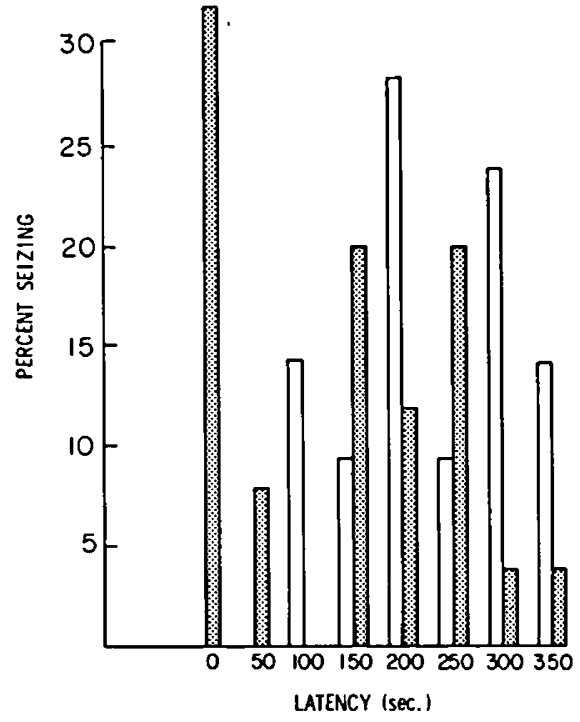

Fig. 5. Latencies (interval between beginning of stimulation and onset of seizure) observed during the 7-month testing period (post-treatment). The bars represent the percentage of all animals seizing that displayed a given latency and are based on the mean for each animal. Each mean latency value actually represents a range of $\pm 25 \mathrm{sec}$. Control, $\square$; phenobarbital, 嘈.

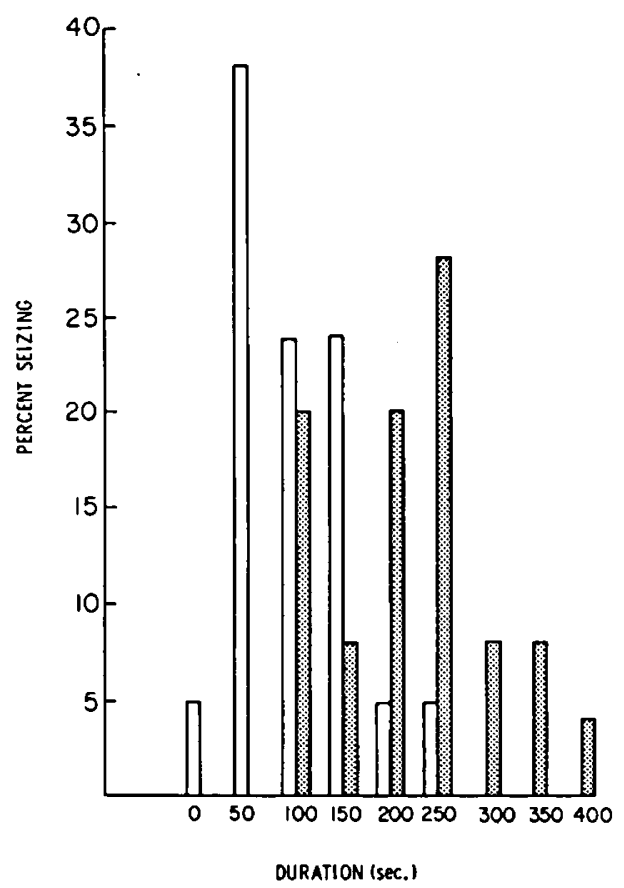

Fig. 6. Duration of seizures elicited during the 7-month post-treatment period. The percentages are based on mean seizure duration times for individual animals. Each time period represents a range of $\pm 25 \mathrm{sec}$. Control, $\square$; phenobarbital, 国.

and greater susceptibility to infections. Four percent of the phenobarbital group (three animals) and 6\% of the controls (four animals) died during the posttreatment period.

\section{DISCUSSION}

Genetically seizure-sensitive Mongolian gerbils exhibit tonicclonic reflex-type epileptic seizures of varying degrees of severity. Seizures can be elicited by handling of the cage or otherwise disturbing or manipulating the animals, but a greater stress is frequently required. The mildest form of seizure is manifested by rapid twitching movements of the whiskers and/or ears. In its most severe form the animal exhibits extreme opisthotonos followed by tonic-clonic movements of the head and limbs. Seizuresusceptible gerbils generally show initial signs of seizure activity from 5-6 weeks of age, although it may occur as early as 4 weeks or as late as 10 weeks. Early seizures are usually very mild, but they become more pronounced as the gerbil matures. A seizuresensitive adult gerbil retains the seizure trait throughout its lifetime.

Based on our own observations and those of others $(5,8)$, phenobarbital in sufficient dosages is an effective anticonvulsant agent in seizure-sensitive adult gerbils. There seems to be little doubt that the drug acts in the expected fashion to prevent or reduce seizure frequency and severity.

The surprising aspect of this study was the evidence that phenobarbital, chronically administered to developing gerbils, actually lowered the seizure threshold and increased seizure intensity. These effects did not disappear upon termination of phenobarbital administration but remained for the duration of the study (6-7 months after discontinuation of phenobarbital). There seems to be little reason to doubt that the drug has in some way modified or altered brain mechanisms involved in the seizure process. Seizures can be elicited more quickly and easily and are of greater intensity than in control animals.

The fact that anticonvulsant drugs may exert convulsant actions under certain circumstances is not unknown. Phenytoin (Dilantin) has been reported to increase seizure activity in humans (6), and withdrawal of multiple anticonvulsants has been reported to result in diminished seizures (4). Reflex epilepsies have been notoriously refractory to anticonvulsant agents of all types. However, these clinical observations have not been studied experimentally, and, to our knowledge, there is no clearcut evidence that anticonvulsants may permanently intensify seizures in developing animals.

The mechanism by which phenobarbital may provoke seizure activity is completely open to speculation. It is of interest to consider the evidence that inhibitory systems in the brain are involved in the maintenance of normal neural activity. In particular, the cerebellum and its projection systems have been associated with inhibitory mechanisms that act to suppress seizure activity in the cerebrum (1). The cerebellum is particularly vulnerable to stressful events occurring in early development (3). One may conceive of prolonged phenobarbital administration interfering with the development of cerebellar inhibitory systems involved in the limitation of seizure activity in the gerbil. Those animals exposed to phenobarbital at critical periods of development may never form, or form incompletely, the inhibitory mechanisms necessary to restrict seizure activity.

There are significant implications to be considered if this explanation should be valid. What might be the consequence of prolonged phenobarbital administration when inhibitory systems are being elaborated in the human infant? Regular phenobarbital

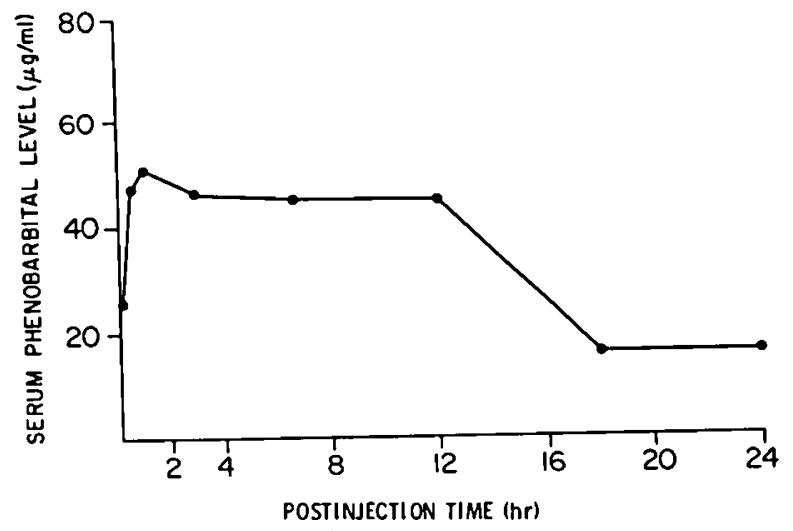

Fig. 7. Serum phenobarbital levels measured at various times following sc injection of phenobarbital $(60 \mathrm{mg} / \mathrm{kg})$ in 31 -day-old gerbils. Each point represents a value obtained from pooled blood (four to six animals). 
administration is often prescribed to human infants who exhibit spontaneous or febrile seizures. Long term controlled evaluations are difficult to carry out in humans and the question remains whether such practices may predispose the human to seizure disorders. Further studies of the apparent convulsant effects of prolonged phenobarbital administration are required.

\section{CONCLUSION}

Chronic administration of $60 \mathrm{mg} / \mathrm{kg}$ phenobarbital to Mongolian gerbils between 1 and 5 months of age intensified seizure activity during the period of treatment. This alteration in seizure behavior was manifested throughout the 7-month period following drug treatment and was therefore assumed to result from a permanent modification(s) in brain mechanisms that relate to seizure activity.

\section{REFERENCES AND NOTES}

1. Cooper, I. S., Beklan, M., and Snider, R. S.: The Cerebellum, Epilepsy and Behavior (Plenum, New York, 1974).

2. Diaz, J., Schain, R. J., and Bailey, B. G.: Phenobarbital-induced brain growth retardation in artificially reared rat pups. Biol. Neonate, 32: 77 (1977).

3. Dobbing, J., Hopewell, J., Lynch, A., and Sands, J.: Vulnerability of developing brain. I. Some lasting effects of x-radiation. Exp. Neurol., 28: 442 (1970).
4. Hansen, R., and Menkes, J.: A new anticonvulsant in the management of minor motor seizures. Devel. Med. Child Neurol., 14: 3 (1972).

5. Kaplan, H. and Miezejeski, C.: Development of seizures in the Mongolian gerbil (Meriones unguiculatus). J. Comp. Physiol. Psychol., 81: 267 (1972).

6. Levy, L. L., and Fenichel, G. M.: Diphenyl hydantoin activated seizures. Neurology, 15: 716 (1965).

7. Livingston, S.: Comprehensive Management of Epilepsy in Infancy, Childhood and Adolescence, p. 174 (Charles C Thomas, Springfield, IL, 1972).

8. Loskota, W. J., and Lomax, P.: The Mongolian gerbil as an animal model for the study of the epilepsies: Anticonvulsant screening. Proc. West. Pharmacol. Soc., 17: 40 (1974).

9. Loskota, W. J., Lomax, P., and Rich, S. J.: The gerbil as a model for the study of the epilepsies: Seizure patterns and ontogenesis. Epilepsia, 15: 109 (1974).

10. Schain, R. J., and Watanabe, K.: Effect of chronic phenobarbital administration upon brain growth of the infant rat. Exp. Neurol., 47: 509 (1975).

11. Schain, R. J., and Watanabe, K.: Origin of brain growth retardation in young rats treated with phenobarbital. Exp. Neurol., 50: 806 (1976)

12. Our colony was developed from 14 Tumblebrook Farm gerbils obtained from Dr. Peter Lomax, Department of Pharmacology, UCLA.

13. Chronic administration of phenobarbital results in accelerated disposition because of the induction of hepatic enzyme systems. Chronically treated animals would be expected to have lower serum levels.

14. This research was supported by USPHS Grants HS-07691, HD-05615, and HD04612.

15. Requests for reprints should be addressed to: Dr. Kathy S. Watanabe, Department of Psychiatry, NPI-MR, University of California, Center for the Health Sciences, Los Angeles, CA 90024 (USA).

16. Received for publication August 2, 1977.

17. Accepted for publication November $2,1977$. 